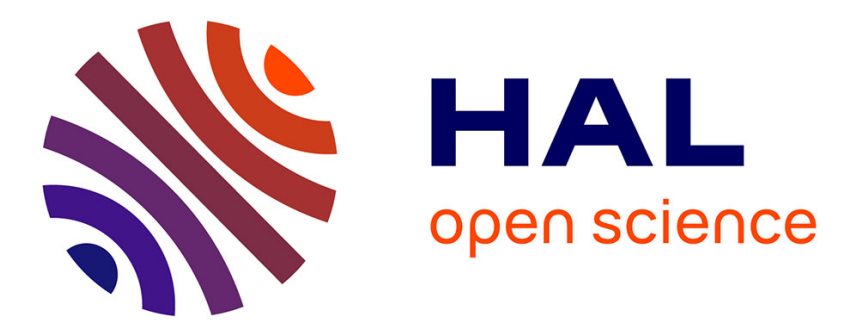

\title{
Computational nonlinear stochastic dynamics with model uncertainties and nonstationary stochastic excitation
}

\author{
Evangéline Capiez-Lernout, Christian Soize, M. P. Mignolet
}

\section{To cite this version:}

Evangéline Capiez-Lernout, Christian Soize, M. P. Mignolet. Computational nonlinear stochastic dynamics with model uncertainties and nonstationary stochastic excitation. ICOSSAR 2013, 11th International Conference on Structural Safety and Reliability, Columbia University, Jun 2013, NewYork, United States. pp.1-8. hal-00806412

\section{HAL Id: hal-00806412 https://hal.science/hal-00806412}

Submitted on 30 Mar 2013

HAL is a multi-disciplinary open access archive for the deposit and dissemination of scientific research documents, whether they are published or not. The documents may come from teaching and research institutions in France or abroad, or from public or private research centers.
L'archive ouverte pluridisciplinaire HAL, est destinée au dépôt et à la diffusion de documents scientifiques de niveau recherche, publiés ou non, émanant des établissements d'enseignement et de recherche français ou étrangers, des laboratoires publics ou privés. 


\title{
Computational Nonlinear Stochastic Dynamics With Model Uncertainties and Nonstationary Stochastic Excitation
}

\author{
E. Capiez-Lernout, C. Soize \\ Laboratoire Modélisation et Simulation Multi-Echelle, MSME UMR 8208 CNRS \\ Université Paris-Est Marne-la-Vallée, Marne-la-Vallée, France
}

M.-P. Mignolet

SEMTE, Faculties of Mechanical and Aerospace Engineering

Arizona State University, Tempe, USA.

\begin{abstract}
The construction of advanced numerical methodologies for the prediction of the dynamical behavior of complex uncertain structures represents an important current challenge. In the present work, structures undergoing large displacements and high strains are investigated. Of particular interest is the analysis of the post-buckling dynamics of a cylindrical shell submitted to an horizontal seismic excitation. The nominal (i.e. without uncertainties) computational model of the cylindrical shell is large, i.e. comprising about 4200000 degrees of freedom, obtained with the finite element method using three-dimensional solid elements. A nonlinear reduced-order modeling is first carried out. Then, model uncertainties (on geometry, material properties, etc.) are introduced using probabilistic methods and the corresponding stochastic reduced-order nonlinear computational model is obtained. The identification of its parameters is next carried out using nonlinear static post-buckling data. Finally, a numerical nonlinear dynamic analysis of the uncertain shell is performed in a seismic context, for which the base of the cylindrical shell is submitted to a prescribed rigid shear displacement, modeled through a centered non-stationary Gaussian second-order stochastic process. The stochastic displacement field is then calculated and the effects of uncertainties and of nonlinearities are analyzed in details.
\end{abstract}

\section{INTRODUCTION}

The paper focuses on the modelling of the postbuckling mechanical behavior of thin cylindrical shells. A discrepancy between experimental measurements and numerical predictions is commonly observed, due to the particular sensitivity of thin cylindrical shells to the presence of initial imperfections (heterogeneity of the materials, imperfect boundary conditions, inhomogeneous thickness induced by the manufacturing process and geometry, etc.). Note for cylindrical shells of very small thickness that the geometrically nonlinear effects induced by large strains and large displacements must be taken into account. Numerous sensitivity analyses to standard geometric imperfections can be found in the literature. However, a generic sensitivity analysis of such structures with respect to a broad class of imperfections requires the introduction of adapted probabilistic approaches to represent uncertainties. Problems involving large nonlinear computational models, taking into account either or both the presence of random uncertainties and the stochastic nature of the loading re- quires appropriate strategies in dynamical analysis, see for instance (Pradlwarter, Schueller, \& Schenk 2003, Pradlwarter \& Schueller 2011). More particularly, nonlinear stochastic buckling analyses have recently been conducted in which geometrical imperfections (Broggi \& Schuëller 2011) and random boundary conditions (Schenk \& Schuëller 2007) were modeled as Gaussian random fields. Such probabilistic models of uncertainties will be referred to as parametric here as they focus the uncertainty only on specific aspects/parameters of the computational models selected by the analyst. An alternative approach, referred to as the nonparametric probabilistic approach, has been developed for situations in which the uncertainty cannot be singled out in one or a few parameters in the computational model. It allows the consideration of both system-parameter uncertainties and model uncertainties (Soize 2012) by proceeding at the level of modal/reduced-order models developed on deterministic bases. Note that the nonparametric approach has been extended to uncertain nonlinear reduced-order models of geometrically nonlinear structures (Mignolet \& Soize 2008). The devel- 
opment of such nonlinear reduced-order models requires first the selection of an appropriate deterministic basis for the representation of the response, see (Mignolet, Przekop, Rizzi, \& Spottswood 2012) for a state-of-the-art on the subject. Having established the reduced-order model of the mean structure, uncertainties on the linear and nonlinear parts of the stiffness operator are introduced in the nonparametric framework. This is accomplished through the construction of a dedicated random operator with values in the set of all positive-definite symmetric real matrices whose mean value involves all linear, quadratic and cubic stiffness terms of the mean nonlinear reducedorder model (Mignolet \& Soize 2008). The resulting stochastic nonlinear computational model is characterized by a single scalar dispersion parameter, quantifying the level of uncertainty in the stiffness properties which can easily be identified with experiments. Experimental validations based on this theory can be found in (Capiez-Lernout, Soize, \& Mignolet 2012, Murthy, Wang, Perez, Mignolet, \& Richter 2012) for slender elastic bodies, e.g. beams. The paper is organized as follows. Section 2 summarizes the main steps leading to the stochastic nonlinear computational model using the nonparametric probabilistic approach for modeling the random uncertainties. A Gaussian non-stationary second-order stochastic process is also introduced to represent the prescribed, earthquake-induced ground-based motions. An identification effort is then carried out to calibrate the stiffness dispersion parameter of the stochastic nonlinear computational model from experimental measurements of the response of the cylindrical shell. Finally, the nonlinear post-buckling dynamical analysis of the uncertain cylindrical shell is carried out using the previously identified stochastic nonlinear computational model subjected to the prescribed ground motions.

\section{FORMULATION OF THE PROBLEM}

\subsection{Mean reduced-order nonlinear computational model}

The cylindrical shell is assumed to be composed of a linear elastic material and to undergo large deformations inducing geometrical nonlinearities. A total Lagrangian formulation is chosen and a mean nonlinear finite element computational model of the structure (with $n \mathrm{DOF}$ ) is formulated using three-dimensional solid finite elements with 8 nodes. In this context, the mean reduced-order nonlinear computational model of the structure is constructed and yields the following set of nonlinear coupled differential equations:

$$
\begin{array}{r}
\mathcal{M}_{\alpha \beta} \ddot{q}_{\beta}+\mathcal{D}_{\alpha \beta} \dot{q}_{\beta}+\mathcal{K}_{\alpha \beta}^{(1)} q_{\beta}+\mathcal{K}_{\alpha \beta \gamma}^{(2)} q_{\beta} q_{\gamma}+\ldots \\
\ldots \mathcal{K}_{\alpha \beta \gamma \delta}^{(3)} q_{\beta} q_{\gamma} q_{\delta}=\mathcal{F}_{\alpha}^{\text {stat }}+\mathcal{F}_{\alpha}^{\text {dyn }}
\end{array}
$$

with initial conditions

$\mathbf{q}(0)=\mathbf{0} \quad, \quad \dot{\mathbf{q}}(0)=\mathbf{0}$

In Eq.(1), $\mathbf{q}=\left(q_{1}, \ldots, q_{N}\right), N \lll n$ is the vector of the generalized coordinates related to the $\mathbb{R}^{n}$-vector of the physical displacements $\mathbf{u}$ by

$\mathbf{u}(t)=\sum_{\beta=1}^{N} \varphi^{\beta} q_{\beta}(t)$,

and the reduced operators $\mathcal{M}_{\alpha \beta}, \mathcal{D}_{\alpha \beta}, \mathcal{K}_{\alpha \beta}^{(1)}$ and $\mathcal{K}_{\alpha \beta \gamma}^{(2)}$, $\mathcal{K}_{\alpha \beta \gamma \delta}^{(3)}$ are related to the mass, damping, linear stiffness terms and to the quadratic, cubic nonlinear stiffness terms whose explicit construction is detailed in the context of solid finite elements in (CapiezLernout, Soize, \& Mignolet 2012). In Eq.(1), the reduced load is split into a static and a dynamic contribution whose latter is issued from a prescribed rigidbody displacement of the base of the cylindrical shell. In Eq.(3), the $\mathbb{R}^{N}$ vectors $\varphi^{\alpha}, \alpha=\{1, \ldots, N\}$ of the projection basis are assumed to be orthonormal such that $\left(\varphi^{\alpha}\right)^{T} \varphi^{\alpha}=\delta_{\alpha \beta}$, in which $\delta_{\alpha \beta}$ is the Kronecker symbol such that $\delta_{\alpha \beta}=1$ if $\alpha=\beta$ and $\delta_{\alpha \beta}=0$ otherwise.. The efficiency of the mean nonlinear computational model strongly depends on the choice of this projection basis as can be reviewed in (Mignolet, Przekop, Rizzi, \& Spottswood 2012). In the present case, the choice of the projection basis is motivated by available experimental data in the nonlinear static post-buckling (Michel, Limam, \& Jullien 2000). For this reason, the projection basis is chosen to be composed of (1) the static POD basis (Proper Orthogonal Decomposition) obtained from the mean nonlinear finite element model and (2) selected linear eigenmodes of vibration issued from the mean linear finite element model. All the details can be found in (Capiez-Lernout, Soize, \& Mignolet 2013).

\subsection{Stochastic reduced-order nonlinear computational model}

In this Section, the nonparametric probabilistic approach is used for modeling the uncertainties in the nonlinear computational model and the dynamic loading represents an earthquake excitation which is modeled by a nonstationary stochastic process.

The main idea of the nonparametric probabilistic approach is to replace each of the reduced-operators of Eq.(1) by random operators defined on the probability space $(\Theta, \mathcal{T}, \mathcal{P})$. The probability model of these random operators is constructed from the maximum entropy principle using the available information (Soize 2005, Soize 2012). More particularly, it has been shown in (Mignolet \& Soize 2008) that the nonparametric probabilistic theory, initially introduced in the linear context for positive-definite symmetric operators, can be extended to the geometrically 
nonlinear context. In this case, it can be shown that the level of uncertainty is quantified by three dispersion parameters $\delta_{M}, \delta_{D}$ and $\delta_{K}$ each being defined on a subset $\Delta_{M}, \Delta_{D}$ and $\Delta_{K}$ of $\mathbb{R}$. Note that the stiffness dispersion parameter $\delta_{K}$ is a single scalar parameter which simultaneously controls the uncertainty level of all the linear and nonlinear stiffness contributions.

The earthquake excitation is issued from a groundbased motion along a given direction. The stochastic dynamical load $\mathcal{F}^{\text {dyna }}(t)$ is modeled by the random vector

$$
\mathcal{F}^{\text {dyna }}(t)=-\left[L_{M}\right]^{T}\left[\mathbf{G}_{M}\left(\delta_{M}\right)\right]\left[L_{T}\right] \boldsymbol{\Gamma}(t),
$$

with values in $\mathbb{R}^{N}$, in which $\left[L_{M}\right]$ is the $(N \times N)$ matrix issued from the Cholesky decomposition of reduced mass operator $[\mathcal{M}],\left[\mathbf{G}_{M}\left(\delta_{M}\right)\right]$ is the random operator with values in the set of the positivedefinite symmetric $(N \times N)$ matrices corresponding to the random germ of the random mass operator, and where the $\mathbb{R}^{N}$-vector $\left[L_{T}\right]$ is related to the direction of the excitation and to the mass data, see (CapiezLernout, Soize, \& Mignolet 2013). In Eq.(4), the acceleration $\{\boldsymbol{\Gamma}(t), t \geq 0\}$ is modeled here by a Gaussian, non-stationary, centered, second-order stochastic process defined on a probability space $\left(\Theta^{\prime}, \mathcal{T}^{\prime}, \mathcal{P}^{\prime}\right)$ which is different from probability space $(\Theta, \mathcal{T}, \mathcal{P})$. Consequently, the stochastic process $\Gamma$ is completely defined by its autocorrelation function $R_{\Gamma}\left(t, t^{\prime}\right)=$ $\mathcal{E}\left\{\boldsymbol{\Gamma}(t) \boldsymbol{\Gamma}\left(t^{\prime}\right)\right\}$. The following usual representation (Kree \& Soize 1986) of $\boldsymbol{\Gamma}$ for earthquake accelerogramms is adopted

$$
\boldsymbol{\Gamma}(t)=g(t) \boldsymbol{\beta}(t),
$$

in which the function $g(t)$ is the envelope function whose representation can be found in (Saragoni \& Hart 1974, Boore 2003). Further, $\{\boldsymbol{\beta}(t), t \in \mathbb{R}\}$ is a real-valued Gaussian, stationary, centered, secondorder stochastic process for which the power spectral density function $S_{\boldsymbol{\beta}}(\omega)$ can be written as a rational function (Kanai 1957). We then have

$$
R_{\boldsymbol{\Gamma}}\left(t, t^{\prime}\right)=g(t) g\left(t^{\prime}\right) R_{\boldsymbol{\beta}}\left(t-t^{\prime}\right),
$$

where $R_{\boldsymbol{\beta}}\left(t-t^{\prime}\right)$ is the autocorrelation function of the stochastic process $\boldsymbol{\beta}(t)$, i.e.

$R_{\boldsymbol{\beta}}\left(t-t^{\prime}\right)=\int_{\mathbb{R}} S_{\boldsymbol{\beta}}(\omega) e^{i \omega\left(t-t^{\prime}\right)} d \omega$

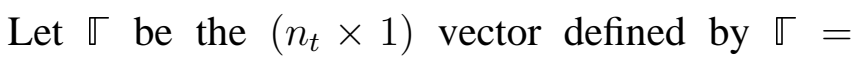
$\left(\Gamma\left(t_{1}\right), \ldots \Gamma\left(t_{n_{t}}\right)\right)$. We introduce the time sampling $t_{i}=(i-1) \Delta t, i=\left\{1, \ldots, n_{t}\right\}$, of $[0, T]$ with $T=$ $n_{t} \Delta t$. Then denote by $\left[R_{\llbracket}\right]=\mathcal{E}\left\{\mathbb{\square} \mathbb{\varpi}^{T}\right\}$ the correla-

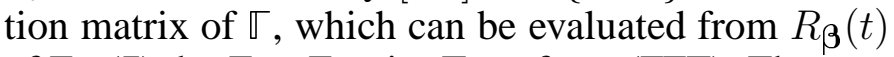
of Eq.(7), by Fast Fourier Transform (FFT). The ran-

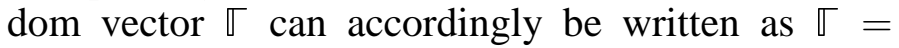

$[L] \mathbf{Z}$, in which $[L]$ is such that $\left[R_{\llbracket}\right]=[L]^{T}[L]$. Further, $\mathbf{Z}=\left(\mathbf{Z}_{1}, \ldots \mathbf{Z}_{n_{t}}\right)$ is a Gaussian random vector such that $\mathcal{E}\left\{\mathbf{Z}_{j}\right\}=0, \mathcal{E}\left\{\mathbf{Z}_{j}^{2}\right\}=1, \mathbf{Z}_{1}, \ldots, \mathbf{Z}_{n_{t}}$ being statistically independent.

The stochastic response is represented by the $\mathbb{R}^{n}$ valued, non-stationary in time, second-order stochastic process $\mathbf{U}(t)$, defined on the product of probability spaces $(\Theta, \mathcal{T}, \mathcal{P})$ and $\left(\Theta^{\prime}, \mathcal{T}^{\prime}, \mathcal{P}^{\prime}\right)$, which is written as

$$
\mathbf{U}(t)=\sum_{\beta=1}^{N} \boldsymbol{\varphi}^{\beta} \mathbf{Q}_{\beta}(t)
$$

in which $\mathbf{Q}=\left(Q_{1}, \ldots, Q_{N}\right), N \lll n$ is solution of the stochastic set of nonlinear differential equations

$$
\begin{array}{r}
\mathcal{M}_{\alpha \beta} \ddot{Q}_{\beta}+\mathcal{D}_{\alpha \beta} \dot{Q}_{\beta}+\mathcal{K}_{\alpha \beta}^{(1)} Q_{\beta}+\mathcal{K}_{\alpha \beta \gamma}^{(2)} Q_{\beta} Q_{\gamma}+\ldots \\
\mathcal{K}_{\alpha \beta \gamma \delta}^{(3)} Q_{\beta} Q_{\gamma} Q_{\delta}=\mathcal{F}_{\alpha}^{\text {stat }}+\mathcal{F}_{\alpha}^{\text {dyn }}
\end{array}
$$

with initial conditions

$\mathbf{Q}(0)=\mathbf{0} \quad, \quad \dot{\mathbf{Q}}(0)=\mathbf{0}$.

With regard to the numerical solver used, for each Monte Carlo realization, a Newmark method is used (Bathe 1982), which employs the averaging acceleration scheme known to be unconditionally stable. With this solver, a set of nonlinear algebraic equations must be solved at each sampling time. This computation is addressed by the fixed-point method or by the Crisfield arc-length method (Crisfield 1997) (the fixedpoint is favored but if not convergent, it is replaced by the Crisfield algorithm).

\section{EXPERIMENTAL IDENTIFICATION IN THE CONTEXT OF STATIC POST-BUCKLING}

This Section is devoted to the experimental validation of the methodology on a thin cylindrical shell for which experimental data is available. Experimental nonlinear static responses are available as a function of the static shear load magnitude (Michel, Combescure, \& Jullien 2000, Michel, Limam, \& Jullien 2000). For comparison with this data, the stochastic computational model is constructed without the mass and the dissipation contributions. Furthermore, the external loading is deterministic.

\subsection{Description of the experiments}

The experimental data used in this paper is briefly summarized below, see (Michel 1997) for complete details. The geometry of the cylindrical shell is characterized by the mean radius $R=0.125 \mathrm{~m}$, thickness 
$e=27010^{-6} \mathrm{~m}$ and height of $0.125 \mathrm{~m}$. It is composed of nickel, which is assumed to be a linear elastic material in the range of stresses considered. The bottom of the cylindrical shell is clamped to a base plate, assumed to be rigid. The top of the cylindrical shell is connected to a rigid plate, of thickness $0.019 \mathrm{~m}$, yielding a total height of the structure $h=0.144 \mathrm{~m}$. The loading is transmitted from servo-cylinders through this rigid plate. A constant traction load $F^{t}=8500 \mathrm{~N}$ is applied to delay the onset of the post-buckling. A controlled static shear point load $F^{s h}$ with maximum magnitude of $9750 \mathrm{~N}$, is then applied at the top of the shell. Let $\left(s_{1}, \cdots, s_{53}\right)$ be the sampling of the increasing static shear load, in which $s_{1}$ corresponds to $0 \mathrm{~N}$ and $s_{53}$ to $9750 \mathrm{~N}$. The displacement corresponding to a given static shear load increment $s_{j}$ is measured at the observation point, which is the point at which the external load is applied. This measured displacement is denoted by $u_{e x p}\left(s_{j}\right)$. Two different mechanical behaviors can be observed on the experimental load-displacement curve : (1) an elastic domain until the critical shear load $F_{\text {crit }}^{\text {sh }}=7450 \mathrm{~N}\left(s_{\text {crit }}=0.745\right)$ at which the displacement is $u_{c r i t}^{\text {exp }}=1.53 \times 10^{-4} \mathrm{~m}$; (2) an approximately linear elastic behaviour in the postbuckling domain investigated.

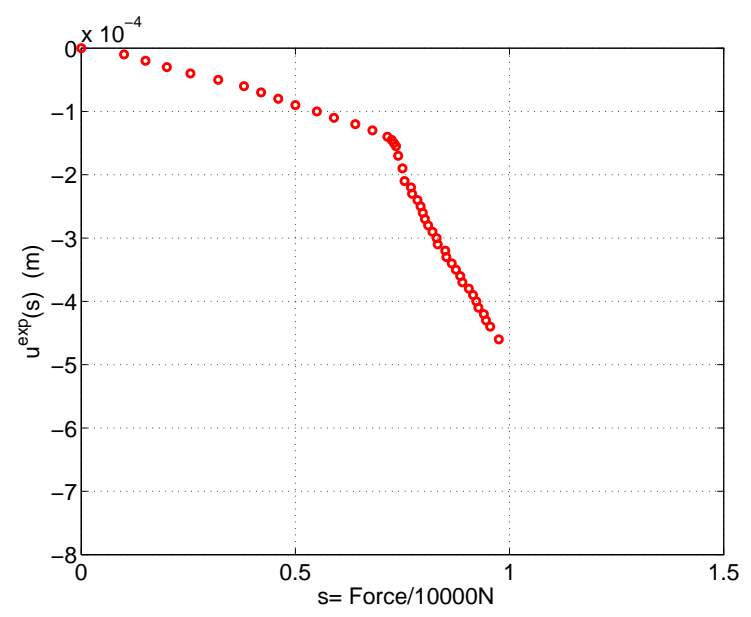

Figure 1: Experimental non-linear response $s \mapsto u^{\exp }(s)$

\subsection{Description of the mean finite element model}

The three-dimensional structure is modeled by a circular cylindrical shell of height $h=0.144 \mathrm{~m}$, mean radius $0.125 \mathrm{~m}$ and thickness $2.7 \times 10^{-4} \mathrm{~m}$. Its bottom is clamped and the upper ring is rigid with three DOFs in translation. The structure is subjected to the external shear point load $F^{s h}$ at $x_{3}=h$, and constant traction point load $F^{t}$ at $x_{3}=h$. The isotropic linear elastic material properties have been experimentally measured to be $E=1.8 \times 10^{11} \mathrm{~N} . \mathrm{m}^{-2}$ and $\nu=0.3$. The mass density is taken as $\rho=8200 \mathrm{Kg} \times \mathrm{m}^{-3}$. It should be noted that a concentrated mass $M^{\prime}=$ $80 \mathrm{Kg}$ is added at the free node located at the top of the rigid plate along direction $\mathbf{e}_{2}$ the direction of the shear point load). However, replacing the external traction load $F^{t}$ by $F^{\prime} t=F^{t}+800 N$ does not change notably the experimental conditions described in the subsection above. The finite element model is a regular mesh composed of $\left(n_{r}-1\right) \times n_{\theta} \times\left(n_{3}-1\right)=$ $1 \times 7500 \times 9599=7125008$-nodes solid finite elements with 8 Gauss integration points. The mean computational model thus has 4230003 degrees of freedom. The observation is the displacement of the node $o b s$, which coincide with the location of the experimental observation, and is denoted by $u_{o b s}(s)$. The first linear elastic buckling mode was computed to occur for a critical shear load $F_{c r i t}=9400 \mathrm{~N}$. Although the experimental critical shear load is found to be $7450 \mathrm{~N}$, the computed buckling mode is in agreement with its experimental counterpart (Michel, Limam, \& Jullien 2000). A sensitivity analysis, conducted with respect to the thickness $e$, the choice of boundary conditions, the parameters $n_{\theta}$ and $n_{3}$ controlling the size of the mesh with the current 8-node finite elements, has shown these computational results to be the closest to the experimental ones. Knowing that the buckling load decreases with respect to initial imperfections, a geometrical perturbation taken as the first linear elastic buckling mode shape is added to the structure with a maximum amplitude of $2.7 \times 10^{-4} \mathrm{~m}$ (Michel, Limam, \& Jullien 2000). It thus allows the buckling to be numerically induced.

\subsection{Construction of the mean nonlinear reduced static computational model}

The nonlinear static response of the finite element model will be referred to the reference solution. Figure 2 shows the graph $s \mapsto u_{o b s}(s)$ for $s$ belonging to $[0,1.5]$ (thick dashed line) which is to be compared with Fig. 1. A quasi-linear elastic response can be observed for $s<0.45$ and then, a smooth transition corresponding to the buckling $(0.45<s<0.85)$. Seen next is a post-buckling with a quasi-linear elastic behavior $(0.85<s<0.975)$ (similarly to Fig.1) until a linear elastic stiffening takes place $(0.975<s<$ 1.5),$s=1.5$ corresponds to a shear point load of $15000 \mathrm{~N}$ ). It should be noted that the smooth transition is directly related to the geometrical perturbation discussed above (Michel, Limam, \& Jullien 2000). The above nonlinear reference solution is then used for determining a projection basis with the Proper Orthogonal Decomposition method (POD-method), which is known to be particularly relevant for nonlinear problems (Sirovich 1987, Sampaio \& Soize 2007). A convergence analysis, carried out in order to select the number $N$ of POD modes which have to be kept in the numerical simulation, yields $N=27$. 


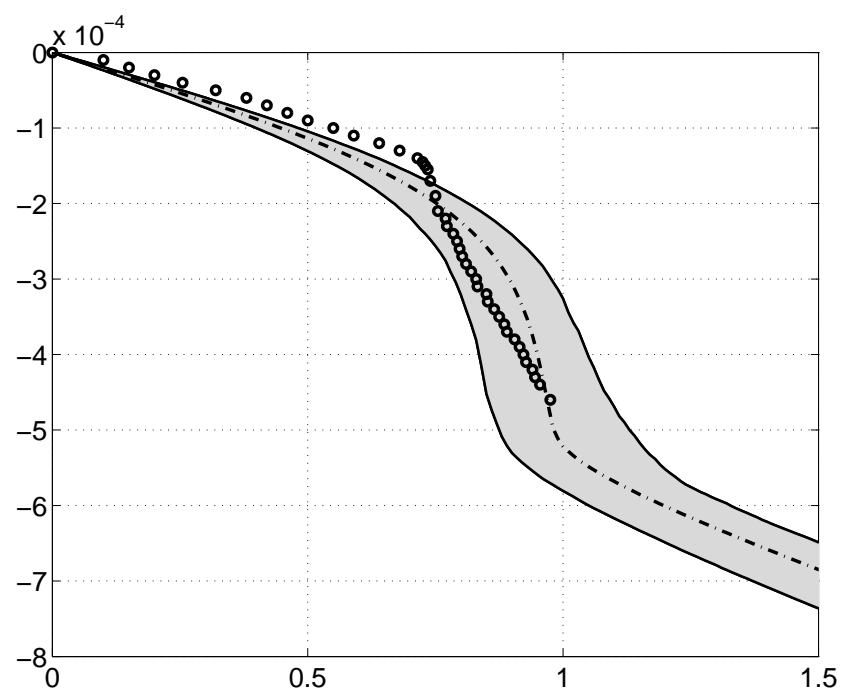

Figure 2: Experimental identification : graph of experimental data $s \mapsto u^{\exp }(s)$ (o markers), graph of the reference response $s \mapsto u^{o b s}(s)$ (thick dashed line), graph of the confidence region of the random response $s \mapsto U_{o b s}(s, \delta=0.45)$ (grey region).

\subsection{Identification of the stochastic nonlinear static computational model}

Since the thin cylindrical shell is very sensitive to small perturbations, a compromise was necessary in the sensitivity analysis mentioned above, between improving the fit of the response in the quasi-linear elastic domain or in the post-buckling domain. A comparison of Figs. 1 and 2 confirms that the nonlinear computational model has the capability to reasonably represent the experimental response. Nevertheless, it remains sufficiently distant from the experiments to justify the implementation of uncertainties leading to a stochastic nonlinear computational model which will be identified using the experimental data. The quasilinear part of the elastic response constructed with the computational model yields acceptable results. The largest discrepancies between computational model and experimental predictions occur in the buckling and post-buckling regimes, it is in these loading conditions that the identification will be performed. This focus is consistent with the physical expectations that the response is most sensitive to uncertainties in these regimes. Based on the above discussion, these observations will be the experimented responses in the interval $s \in[0.75,0.975]$ which is sampled in 34 points denoted by $s_{i}, i=20 \ldots, 53$.

The construction of the stochastic nonlinear reduced-order computational model using the nonparametric probabilistic approach is performed as explained previously. Let $U_{o b s}(s, \delta)$ be the random observations with which the parameter $\delta_{K}$ will be identified. In the present research, the identification procedure is achieved using the maximum likelihood method associated with a statistical reduction of the information (Soize, Capiez-Lernout, Durand, Fernandez, \& Gagliardini 2008). Note that it is particularly adapted to the present case for which the random variables $U_{o b s}\left(s_{i}, \delta\right)$ are not only dependent but also

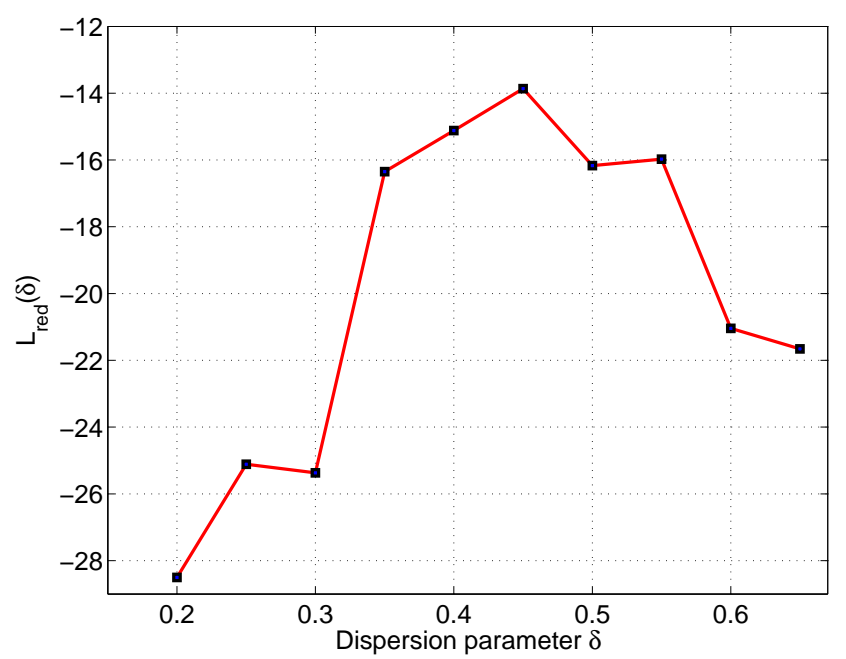

Figure 3: Graph of maximum likelihood function $\delta \mapsto \mathcal{L}^{\text {red }}(\delta)$.

strongly correlated. This approach proceeds with a principal component analysis of the random observations which leads to a reduced set of uncorrelated random variables. The likelihood function, $\delta \mapsto \mathcal{L}^{\text {red }}(\delta)$, is evaluated in the space of these uncorrelated random variables representing the data. It is then obtained with a reduction to 5 uncorrelated random variables and for $\delta \in \Delta=[0.2,0.65]$. It was found that the use of 5 uncorrelated variables yields a relative error of $5.41 \%$ on the trace of the covariance matrix, which was deemed to be sufficiently small. Figure 3 displaying the graph of the likelihood function $\delta \mapsto \mathcal{L}^{\text {red }}(\delta)$, was obtained using the Monte Carlo numerical simulation with $n_{s}=10000$ independent realizations. Note that the likelihood function is replaced by an approximation which is constructed as the product of marginal probability density functions of each uncorrelated random variable. Although this assumption modifies the Likelihood function, it has been shown that its combination with the statistical reduction yield accurate estimations (Soize, Capiez-Lernout, Durand, Fernandez, \& Gagliardini 2008). Seeking the maximum of $\mathcal{L}^{r e d}(\delta)$ for the experimental identification yields the optimal value $\delta^{\text {opt }}=0.45$.

\subsection{Confidence region analysis}

The confidence region of the static nonlinear response predicted with the stochastic nonlinear reduced-order model is then computed with a probability level $P_{c}=$ 0.95; see Figure 2. Also shown on this figure is the experimental nonlinear response. Despite the slight underestimation provided by the optimal stochastic nonlinear computational model in the linear range (induced by the choice of the mean nonlinear computational model as explained above), the results are broadly consistent with the experimental nonlinear response, validating the stochastic nonlinear reducedorder model and its identification. Analyzing the features of the confidence region shown in Fig. 2, it is first observed that the effects of uncertainties increase with the nonlinearity as may be expected. In the buck- 
ling and post-buckling range, for which the static load increment $s$ belongs to $[0.45,0.975]$, the confidence region quickly broadens, allowing some realizations of the random displacement to be almost doubled of others. Consider next the elastic stiffening range, corresponding to a static load increment $s>0.975$, for which no experimental result is available but which can be predicted by the stochastic nonlinear computational model. First, it is seen that the mechanical behavior tends to be a linear one. Moreover, the confidence region is more narrow in this range than in the previous one, which demonstrates a robustness of the random response with respect to both model and system-parameter uncertainties.

\section{DYNAMIC POST-BUCKLING ANALYSIS OF THE CYLINDRICAL SHELL UNDER A SEISMIC GROUND-BASED MOTION}

This section concerns the dynamical analysis of the cylindrical shell subjected to an earthquake basedground motion. The stochastic nonlinear computational model of the shell constructed and identified in the previous Section is reconvened with $\delta_{K}=\delta_{K}^{o p t}=$ 0.45 . Its random operators are defined on the probability space $(\Theta, \mathcal{T}, \mathcal{P})$. Uncertainties on the mass and damping are neglected here but could easily be introduced. The dynamic loading conditions consist in a seismic horizontal motion, applied to the base of the cylindrical shell. The mass being deterministic, the corresponding shear load is a stochastic process indexed by $\mathbb{R}^{+}$and defined on the probability space $\left(\Theta^{\prime}, \mathcal{T}^{\prime}, \mathcal{P}^{\prime}\right)$. Consequently, the stochastic physical response is modeled by $\mathbb{R}^{n}$-random nonlinear stochastic process $\left\{\mathbf{U}(t), t \in \mathbb{R}^{+}\right\}$defined on the product of probability spaces $(\Theta, \mathcal{T}, \mathcal{P})$ and $\left(\Theta^{\prime}, \mathcal{T}^{\prime}, \mathcal{P}^{\prime}\right)$.

\subsection{Definition of the stochastic excitation and of the frequency band of analysis}

The stochastic excitation is simulated as explained in Section 2. For the present application, the power spectral density $S_{\beta}(\omega)$ of the stochastic process $\boldsymbol{\beta}(t)$ is chosen as

$$
S_{\beta}(\omega)=\bar{s} \frac{\bar{\omega}^{4}+4 \bar{\xi}^{2} \bar{\omega}^{2} \omega^{2}}{\left(\omega^{2}-\bar{\omega}^{2}\right)^{2}+4 \bar{\xi}^{2} \bar{\omega}^{2} \omega^{2}},
$$

in which $\bar{s}=1.15 \times 10^{-4} \mathrm{~m}^{2} . \mathrm{s}^{-3}, \bar{\omega}=785.4 \mathrm{rad} . \mathrm{s}^{-1}$ and $\bar{\xi}=0.02$. The frequency band of analysis is $\mathbb{B}_{\nu}=$ $[0,160] H z$. The time sampling is $\delta t=5 \times 10^{-4} s$, the total time duration $T=1.27 \mathrm{~s}$ and therefore, the number of time steps is $n_{t}=2546$. The frequency resolution is $\delta \nu=0.78 \mathrm{~Hz}$ corresponding to a frequency band $[0,1000] \mathrm{Hz}$ (the sampling frequency is $\nu_{e}=2000 \mathrm{~Hz}$ ). The envelope function is a piecewise continuous function defined by $g(t)=25 t^{2} / 4$ if $t<0.4 \mathrm{~s}, g(t)=1$ if $t \in[0.4,0.7] \mathrm{s}$ and $g(t)=$ $e^{-10(t-0.7)}$ if $t>0.7 \mathrm{~s}$. The stochastic excitation is then obtained using Eq.(4).

\subsection{Construction of the mean nonlinear reduced-order computational model}

The static POD projection basis obtained in Section 3 is reused for the present nonlinear dynamic case. Since the convergence rate of the solution constructed with such a projection basis strongly depends on the external applied loads, it is appropriate only if a similar loading class is used. With the presence of the concentrated mass $M^{\prime}$ at the top of the structure, the shear load induced by the ground-based motion is largest at the top of the structure. This excitation, in addition to the external constant traction load, is thus compatible with the loading used for the nonlinear static case. Note that the above statically derived basis is not expected to be sufficient to describe the nonlinear dynamical response of the shell; it needs to be completed by additional basis vectors that we select as linear elastic modes. A usual modal analysis of the linearized dynamical computational model with the predeformation discussed in Section 3 is then performed. An analysis of the first 40 eigenfrequencies and mode reveals that the first two eigenmodes describe global bending modes of the structure. Further, a high modal density is observed, starting with eigenfrequency $\nu_{3}=897.01 \mathrm{~Hz}$. There are 38 local elastic modes belonging to frequency band $[897,1123] \mathrm{Hz}$. The ground motions characterized by the power spectral density of Eq.(11) would strongly excite the first two elastic modes but only weakly the ensuing ones. Thus the projection basis of the stochastic nonlinear reduced-order model must closely represent the first two elastic modes but not necessarily the ensuing ones. As a consequence, these two elastic modes are selected in order to enrich the static POD basis (using a partial Gram-Schmidt orthonormalization procedure). The mean reduced-order computational model is then obtained using the enriched projection basis. The reduced dissipation matrix is constructed according to $[\mathcal{D}]=\zeta[\mathcal{K}]$ by choosing $\zeta=4.44 \times 10^{-5}$. This leads to a critical dissipation rate $\xi \simeq 0.017$ for the two first elastic modes.

\subsection{Results}

The response of the shell is monitored as the same position and in the same direction than above, see Section 4.2. This random observation, denoted by $U_{o b s}(t)$, is a stochastic process indexed by $[0, T]$ and defined on the product of probability spaces $(\Theta, \mathcal{T}, \mathcal{P})$ and $\left(\Theta^{\prime}, \mathcal{T}^{\prime}, \mathcal{P}^{\prime}\right)$. It can then be written as

$U_{o b s}(t)=\underline{u}_{o b s}(t)+U_{o b s}^{c}(t)$,

in which $\underline{u}_{o b s}(t)$ is the deterministic function characterizing the mean of the stochastic nonlinear dynami- 
cal response i.e.

$\underline{u}_{o b s}(t)=\int_{\Theta} \int_{\Theta^{\prime}} U_{o b s}\left(t, \theta, \theta^{\prime}\right) d \mathcal{P}(\theta) d \mathcal{P}^{\prime}\left(\theta^{\prime}\right)$

and where $U_{o b s}^{c}(t)$ is a centered non-stationary stochastic process. In the sequel, $U_{o b s}\left(t, \theta, \theta^{\prime}\right)$ is denoted by $U_{\text {obs }}(t ; \theta)$, where $\theta=\left(\theta, \theta^{\prime}\right)$.

Four analysis cases (see Table 1 ) are investigated, analyzed and compared.

Table 1: Description of the analysis cases

\begin{tabular}{|l|c|c|c|}
\hline & Stiffness & Stiffness & External load \\
\hline Case 1 & linear & deterministic & stochastic \\
\hline Case 2 & linear & stochastic & stochastic \\
\hline Case 3 & nonlinear & deterministic & stochastic \\
\hline Case 4 & nonlinear & stochastic & stochastic \\
\hline
\end{tabular}

Figure 4 displays the graphs of $t \mapsto U_{o b s}^{c}(t, \theta)$ (red line) and $t \mapsto \underline{u}_{o b s}(t)$ (blue line) for the four analysis cases and for a specific realization of the ground motions. It is seen that $\underline{u}_{o b s}(t)$ is a centered oscillating function for the linear cases as expected given the zero mean character of the excitation. However, a small negative mean is observed for the nonlinear cases. Superimposing all cases, it is seen, for this present realization, that the geometric nonlinear effects occur first at $t_{\text {nonlin }}=0.16 \mathrm{~s}$ for a displacement level greater than $1.8 \times 10^{-4} \mathrm{~m}$, or $\frac{2}{3}$ of the shell thickness. Note that these observations are coherent with the results obtained in the static case and shown in Figure 2. Comparing cases 1 and 2 with cases 3 and 4 respectively, it is seen that the effect of the geometrical nonlinearities is to decrease the intensity of the response. Moreover, this effect increases with the presence of model and system-parameter uncertainties in the stochastic computational model. Comparing case 1 with case 2 , it is seen that the presence of random uncertainties significantly spreads the stochastic linear response allowing displacements of $1.7375 \times 10^{-3} \mathrm{~m}$, or 6 thicknesses, to occur. A similar conclusion does not seem to hold in the nonlinear case : when comparing case 3 with case 4 , the presence of random uncertainties only seems to slightly modify the nonlinear dynamical response. Let $t_{0}=0.6695 \mathrm{~s}$, the time for which the response magnitude $U_{o b s}^{c}(t, \mathbb{\theta})$ is maximum. For all cases, Table 2 summarizes the response magnitude corresponding to the closest local maximum of $U_{o b s}^{c}(t, \theta)$ occurring near $t_{0}$. Figure 5 displays the 3D representation of the response of realization $\theta$ corresponding to the local maxima described in Table 2. It is seen that all cases yield different mechanical behaviour. In particular, for case 3, the investigated local maxima occurs in a local region for which the local maxima are inhibited, due to the nonlinear restoring forces induced by the nonlinearity. Moreover, it is seen that the nonlinear cases (case 3 and 4) yield responses which are less spatially smooth compared to the linear cases 1 and 2. It can also be ob-
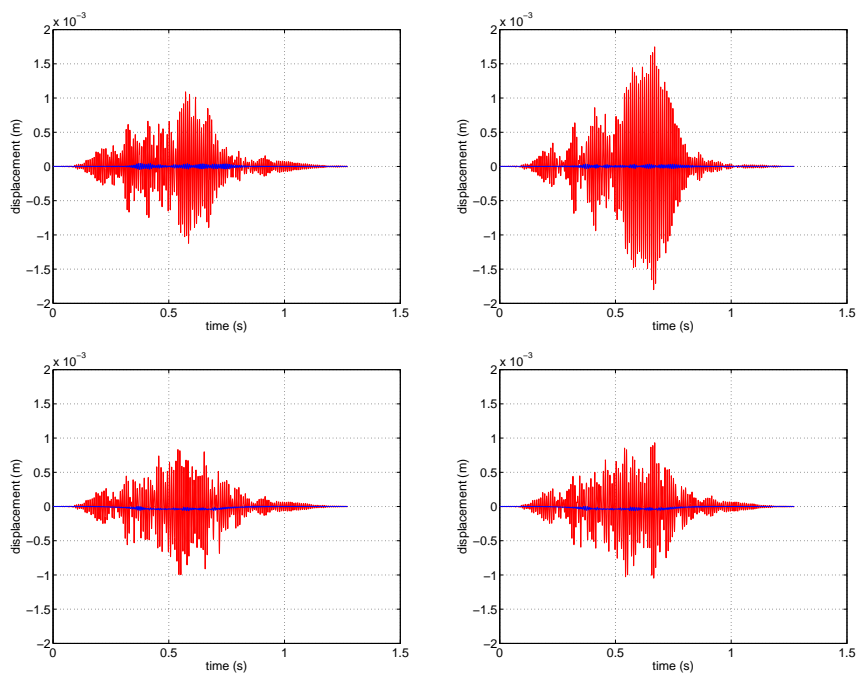

Figure 4: Graphs of functions $t \mapsto U_{o b s}^{c}(t, \Theta)$ (red line) and $t \mapsto$ $\underline{u}_{o b s}(t)$ (blue line) for cases 1 and 2 (upper graphs) and for cases 3 and 4 (lower graphs)

served that for the linear cases, only one global bending elastic mode is excited, whereas the nonlinearities couple the two global bending elastic modes.

Table 2: Description of the local maximum investigated

\begin{tabular}{|c|c|c|}
\hline & Local maximum $(m)$ & time $(s)$ \\
\hline Case 1 & $0.822410^{-3}$ & 0.6685 \\
\hline Case 2 & $1.737510^{-3}$ & 0.6695 \\
\hline Case 3 & $0.374410^{-3}$ & 0.6705 \\
\hline Case 4 & $0.894810^{-3}$ & 0.6695 \\
\hline
\end{tabular}

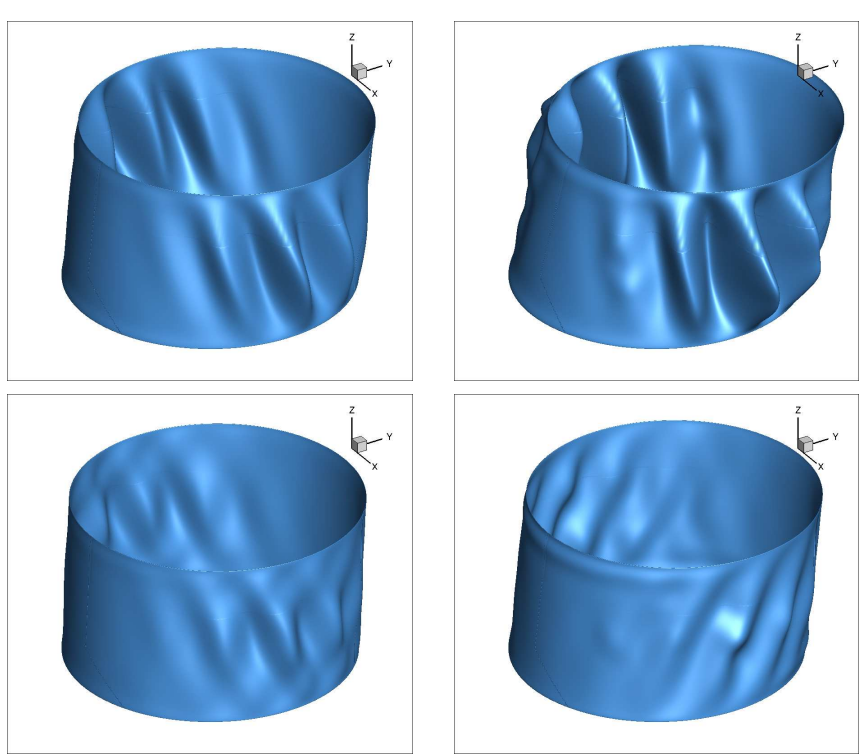

Figure 5: 3D Representation of the response of realization $\theta$ for the cases 1 and 2 (upper graphs) and for cases 3 and 4 (lower graphs)

\section{CONCLUSION}

The paper has presented an experimental validation of an advanced computational method for analyzing the nonlinear post-buckling behavior of a geometrically nonlinear thin shell structure in presence of uncertainties. The experimental static nonlinear response of a very thin cylindrical shell with respect 
to the intensity of a static shear load constitute the experimental data. First, the mean nonlinear computational model has been used for generating the nonlinear static reference response from which the POD basis has been constructed. The nonlinear stochastic computational model has then been constructed and experimentally identified to capture the reference response at best. For this type of very thin structures investigated, the sensitivity of the mechanical behavior is very high. The stochastic modeling is considered appropriate when the experimental response belongs to the confidence region. It was observed that the experimentally identified computational model yields a slight overestimation of the static nonlinear response in the linear range, while the buckling and the postbuckling ranges, which are particularly sensitive to uncertainties, are accurately predicted by the identified stochastic computational model. By increasing the shear load, it has been shown that the computational model predicts a range for which a linear elastic stiffening is observed, for which the robustness to uncertainties lightly increases. Finally, the identified stochastic computational model has been used for predicting the nonlinear dynamical post-buckling response, under a stochastic excitation induced by a ground-based motion of the structure.

\section{REFERENCES}

Bathe, K. (1982). Finite Element Procedures in Engineering Analysis. Prentice-Hall.

Boore, D. (2003). Simulation of ground motion using the stochastic method. Pure and Applied Geophysics 160(3-4), 635-676.

Broggi, M. \& G. Schuëller (2011). Efficient modeling of imperfections for buckling analysis of composite cylindrical shells. Engineering Structures 33, 1796-1806.

Capiez-Lernout, E., C. Soize, \& M.-P. Mignolet (2012). Computational stochastic statics of an uncertain curved structure with geometrical nonlinearity in three-dimensional elasticity. Computational Mechanics 49(1), 87-97.

Capiez-Lernout, E., C. Soize, \& M.-P. Mignolet (2013). Nonlinear stochastic dynamical post-buckling analysis of uncertain cylindrical shells. In 11th International Conference on Recent Advances in Structural Dynamics, RASD 2013, Pisa (Italia), July 1-3, 2013.

Crisfield, M. (1997). Non-linear finite element analysis of solids and structures, Vol. 1 : essentials. John Wiley and Sons, Chichester.

Kanai, H. (1957). Semi-empirical formula for the seismic characteristics of the ground motion. Bulletin of the Earthquake Research Institute 35, 309-325.

Kree, P. \& C. Soize (1986). Mathematics of random phenomena random vibrations of mechanical structures. D. Reidel Pub. Co.

Michel, G. (1997). Flambage de coques minces cylindriques sous un chargement dynamique de cisaillement. $\mathrm{Ph}$. $\mathrm{D}$. thesis, INSA Lyon.

Michel, G., A. Combescure, \& J.-F. Jullien (2000). Finite element simulation of dynamic buckling of cylinders subjected to periodic shear. Thin-Walled Structures 36, 111-135.

Michel, G., A. Limam, \& J.-F. Jullien (2000). Buckling of cylindrical shells under static and dynamic shear loading. Engineering Structures 22, 535-543.

Mignolet, M.-P., A. Przekop, S. Rizzi, \& S. Spottswood
(2012). A review of indirect/non-intrusive reduced order modeling of nonlinear geometric structures. Journal of Sound and Vibration, accepted for publication, http://dx.doi.org/10.1016/j.jsv.2012.10.017.

Mignolet, M.-P. \& C. Soize (2008). Stochastic reduced order models for uncertain geometrically nonlinear dynamical systems. Computer Methods in Applied Mechanics and Engineering 197, 3951-3963.

Murthy, R., X. Wang, R. Perez, M.-P. Mignolet, \& L. Richter (2012). Uncertainty-based experimental validation of nonlinear reduced order models. Journal of Sound and Vibration 331.

Pradlwarter, H., G. Schueller, \& C. Schenk (2003). A computational procedure to estimate the stochastic dynamic response of large non-linear FE-models. Computer Methods in Applied Mechanics and Engineering 192(7-8), 777-801.

Pradlwarter, H. J. \& G. I. Schueller (2011). Reliability of deterministic non-linear systems subjected to stochastic dynamic excitation. International Journal for Numerical Methods in Engineering 85(9), 1160-1176.

Sampaio, R. \& C. Soize (2007). Remarks on the efficiency of pod for model reduction in non-linear dynamics of continuous elastic systems. International Journal for Numerical Methods in Engineering 72(1), 22-45.

Saragoni, G. \& G. Hart (1974). Simulation of artificial earthquakes. Earthquake Engineering and Structural Dynamics 2(3), 249-267.

Schenk, C. \& G. Schuëller (2007). Buckling analysis of cylindrical shells with cutouts including random boundary and geometric imperfections. Computer Methods in Applied Mechanics and Engineering 196(35-36), 3424-3434.

Sirovich, L. (1987, OCT). Turbulence and the dynamics of coherent structures . Quarterly of Applied Mathematics 45(3), $561-571$.

Soize, C. (2005). Random matrix theory for modeling random uncertainties in computational mechanics. Computer Methods in Applied Mechanics and Engineering 194(12-16), 1333-1366.

Soize, C. (2012). Stochastic Models of Uncertainties in Computational Mechanics, Lecture Notes in Engineering Mechanics 2. American Society of Civil Engineers (ASCE).

Soize, C., E. Capiez-Lernout, J.-F. Durand, C. Fernandez, \& L. Gagliardini (2008). Probabilistic model identification of uncertainties in computational models for dynamical systems and experimental validation. Computer Mechanical Methods in Applied Mechanics and Engineering 98(1), 150-163. 